From observations which I have recently made it seems evident to me that the cause for seeing the motion is entirely different.

In the first place, you can always see the motion a fraction of a second before you begin to feel it. In the second place, you cannot see a perfectly horizontal motion or a gentle vertical (heaving) motion. In the third place, watching a fixed point close to you, such as a pattern on a carpet, when the ship is pitching and rolling, is far more tiring to the eyesight than when the ship is motionless or running perfectly steadily. All this points to the appearance being due to a true relative motion of the eyes to the ship.

The eyes are suspended in their muscular settings, much in the same way as are ships' compasses in their binnacles. The eyes are, furthermore, perfectly balanced, so as to make their muscular displacements as little tiring as possible. In their normal position, the pull of gravity is exerted vertically through their centres, and the muscular mechanism is compensated for gravity.

Any angular change of position will displace the eyes just as it displaces the stomach, excepting that the eyes, being a great deal more sensitively suspended, will register the displacements more quickly. It is not, however, the motion of the eyes which strains the eyesight, but the act of resisting this motion.

If, with your eyes shut, you attempt to fix the mental representation of a point, which a moment previously you were watching with eyes wide open, you will find that, after one or two motions of the ship, the bodily feeling will precede any visual sensation which your imagination can conjure up. The imaginary point is no longer fixed, but follows the eyes as they let themselves go to the motions of the ship. No strain of the eyesight is caused by a muscular resistance, and the displacements, while felt, can no longer be seen.

Pittsburg, U.S.A., February 26.

\section{Production of an Electrically Conductive Glass.}

EXPERIMENTs have from time to time been made, both in England and abroad, to ascertain what ingredients are best for the purpose of producing glasses of very high electrical resistance.

The utility of a vitreous substance which would conduct electricity comparatively well does not appear, however, to have so far claimed any consideration.

I beg therefore to direct attention to a glass which has recently been made in my laboratory. Its chief feature is that it readily conducts electricity.

For the windows or cases of electroscopes and all hightension apparatus requiring a transparent cover capable of screening off external electrical fields, this material offers many advantages. A conducting varnish is no longer required for glass which conducts electricity itself. In addition to these practical considerations, there arises the interesting question as to the process by which electricity passes through this substance-whether it is electrolytic. Its resistance varies very markedly with temperature changes. I hope later to give more precise details. The basis of the glass is sodium silicate.

Shooters Hill, Kent, March 12.

Charles E. S. Phillips.

\section{Interpretation of Meteorological Records.}

IN discussing the records of the meteorological instruments at Canterbury (Nature, March 15), Dr. Aitken suggests that the heavy rain which fell dragged down the higher air, and so caused the fall of $12^{\circ}$ indicated on the thermograph curve, and he very clearly and convincingly shows the consequent effect on the barometric pressure and wind velocity. If, however, the air had been in a state of stable equilibrium previous to the thunderstorm, the effect of such a mechanical dragging down of the higher air would have been to heat by compression that air so much that the temperature would have been raised rather than lowered at the ground-level. But if, previous to the storm, the upper air had from any cause become very much colder than the lower air, the atmosphere would be in a state of unstable equilibrium, that is to say, the rate of

vo. I 90J, roL. $7 \hat{3}]$ change of temperature with height would be greater than the adiabatic rate of change due to heating by compression of descending air. In such a case the changes recorded by the various curves may have been initiated by this heavy cold air suddenly descending and displacing the lower air, which by its sudden uprising would be cooled, the moisture in it condensed, and a heavy fall of rain caused.

The lightning which accompanied this storm introduces an element of uncertainty into any attempted explanation, for we do not know yet the manner in which electric charges are generated in the atmosphere. But it seems probable that a great cooling of the higher air is an accompaniment of a state of electric tension, for it is difficult to see otherwise why a thunderstorm should be followed by a lowering of the temperature near the groundlevel.

R. T. OMOND.

Edinburgh.

\section{Oscillation of Flame Cones.}

I SHOULD be glad if any of your readers could give an explanation of the cause of the following flame phenomenon, produced while experimenting with a modification of Prof. Smithells's apparatus for the separation of the cones of a Bunsen flame.

A mixture of gas and air is burned at the top of a vertical tube (made preferably of combustion tubing) about 4 feet long and $\frac{7}{8}$ inch to $I$ inch in diameter, having a delicate screw adjustment for regulating the proportions of gas and air.

The air supply is carefully and slowly increased, until an almost explosive mixture is reached, and the inner cone is very short and sharp and of a light green colour. On admitting a very slight increase of air after this point, the inner cone (sometimes the two cones) descends the tube to a distance of about 2 feet, and then pauses and goes up again, re-joining the outer cone. The flame then "sharpens" again and repeats the process, and will continue to do so for several hours without further adjustment of the gas or air being made.

There is every appearance of an explosion wave being propagated, as shown by the increasing velocity of the descending flame and by the occasional emission of a note as it reaches the end of the travel.

The length of travel can be regulated by the amount of air admitted, varying from I or 2 inches to about 2 feet in the same tube. If it be allowed to exceed a certain limit the inner cone is extinguished at its lowest point, but immediately re-lights at the top of the tube, and then returns as before. The periodicity can be varied from about once in five seconds to once per second.

The gas pressure does not need any special regulation, the ordinary variations from a town supply not affecting the results.

The following are the points requiring explanation:-

(r) As the proportions of gas and air are constant, what is the cause of the periodic "sharpening" of the cones after meeting at the top of the tube?

(2) What prevents the explosion wave being completed, and the consequent firing back of the mixture?

(3) What causes the inner cone to return and travel up the tube, re-joining the outer one at the top?

(4) The alteration in the character of the flame (in view of the fact that the proportions and pressure of gas and air are constant) points to some form of wave motion bringing the molecules into closer contact. If this be so, what are the conditions which set up this wave motion and what determines its periodicity?

Olton, Warwickshire.

Harold E. Temple.

THE phenomenon described in the foregoing letter is in part dealt with in a paper by Dr. Ingle and myself in the Transactions of the Chemical Society for 1892 (vol. 1xi., p. 204). The continued oscillation of the inner cone is, I think, explained by the fact that the mixture of gas and air in the tube is not uniform. We have, indeed, found it necessary to use elaborate mixing appliances to make it uniform. When a portion of the mixture rich in air reaches 